\title{
Research on Monitoring Location Model Based on Geo-Video Semantics
}

\author{
Li Siyi a,b, , Li Wenjing a,b, Hu Dan a,b, * \\ ${ }^{a}$ School of Resource and Environmental Engineering, Wuhan University of Science and Technology, \\ lisiyiame@163.com, wenjingli@wust.edu.cn,danchun1005@hotmail.com \\ ${ }^{b}$ Industrial Safety Engineering Technology Research Center of Hubei Province \\ * Corresponding author
}

Keywords: Geo-video; Monitoring site selection; Horizon

\begin{abstract}
:
With the flourishing and advancement of Smart City, security concerns arise, and the use of video surveillance system is regarded as a means of resolving security problems. The public environment of smart city shows the characteristics of multiscale and spatiotemporal, while Geo-video system has gradually become a trend solution in the monitoring field, which supports the correlation analysis of geo-video data, video content and geographical environment. But it has such a problem of the mass geographic video data: inefficient store, retrieval and linkage analysis. Therefore, Geo-video system has put forward an urgent demand for geographic video data with higher quality and lower scrap rate.
\end{abstract}

The collection of geographic video data still depends on the monitoring equipment, and the picture content depends on the geographical location, frame size, frame rate, resolution, elevation and other parameters of the monitoring equipment. Therefore, the geo-video semantics are taken into account in the monitoring site selection model, and the semantic information such as the characteristics, behaviors and events of the monitoring objects are integrated into the construction of the monitoring screen, which is conducive to the event inference, information mining and correlation analysis of geo-video, and responds to the monitoring target and demand of geo-video system. In this way, it can start from the site selection and serve the overall construction of geo-video system.

Considering the research hotspot and trend in the field of geo-video, we comprehensively analysed the location requirements, monitoring equipment parameters, location semantics and feature semantics of monitoring objects from the perspective of GIS, and a monitoring location model based on the semantics of geo-video has been proposed, which realized the fusion of monitoring picture and 2D map. Firstly, four typical geographic video semantic models (geographic entity, geographic scene, monitoring equipment and location principle) are analyzed to extract a set of extensible geographic video semantic classification model for monitoring location. Secondly, the geographic video semantic model is studied in combination with the research area, including monitoring requirement analysis, classification of geographic scenes and geographic entities, and description of the research area in terms of feature semantics and location semantics. Finally, generate the overlapping facets according to the monitoring candidate group and the visual field of the monitoring equipment, which are input as parameters into the set coverage model based on monitoring location. Linear programming method is used to solve the set coverage problem of regional monitoring scope, and the results are visualized in $2 \mathrm{D}$ or $3 \mathrm{D}$.

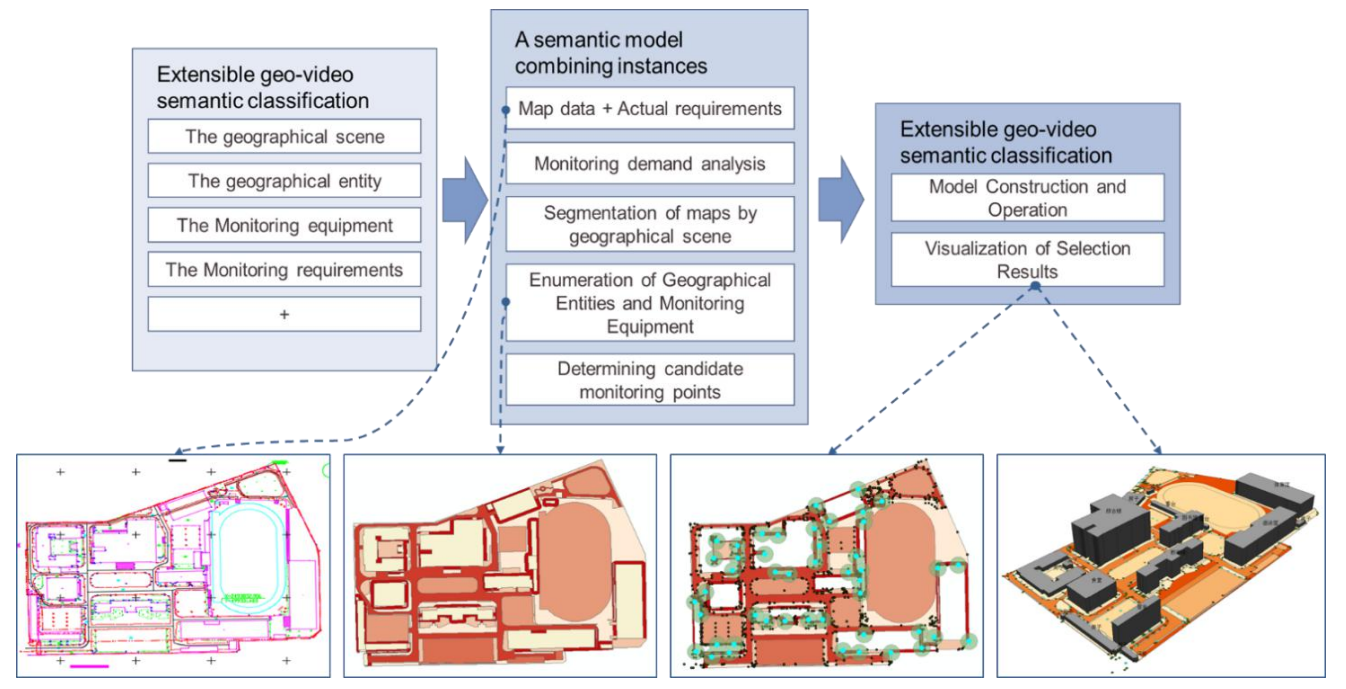

Figure 1. Research Route 
By comparing the two cases of the same method with different data and the same method with different data, it can be found that the location method based on geo-video semantics can scientifically and reasonably select the location of monitoring equipment in the research area, and changes across data and actual situation do not negatively impact the experimental results. The monitoring location method based on geo-video semantics is prospective and stable, and can overcome the problems of uncertain location results, uneven distribution and irregularity in general methods. Monitoring location model based on geo-video semantics can provide high quality geo-video data for video surveillance system and lay a foundation for security construction of smart city. 